\title{
Atmospheric dispersion modeling to simulate rocket exhaust clouds
}

\author{
E. G. S. Nascimento ${ }^{1}$, D. M. Moreira ${ }^{1,2}$, T. T. A. Albuquerque ${ }^{1}$, \\ N. C. Reis Jr ${ }^{1}$, J. M. Santos ${ }^{1}$, E. V. Goulart ${ }^{1} \&$ G. Fisch ${ }^{3}$ \\ ${ }^{I}$ Federal University of Espirito Santo, UFES/PPGEA, Brazil \\ ${ }^{2}$ Center for Integrated Manufacturing and Technology, \\ SENAI/CIMATEC, Brazil \\ ${ }^{3}$ Institute of Aeronautics and Space, IAE, Brazil
}

\begin{abstract}
This work shows the development of a model for the simulation of rocket exhaust effluent. The model is based on a semi-analytical solution of the time dependent three-dimensional advection-diffusion equation and overcomes the features of the Gaussian concepts considering realistic eddy diffusivities and wind profiles. We report numerical simulations with the approach using micrometeorological parameters and wind profile generated by the Weather Research and Forecasting (WRF) model in the area around the Alcântara Launch Center, Brazil.
\end{abstract}

Keywords: Alcântara Launch Center, rocket exhaust clouds, atmospheric dispersion, mathematical modelling.

\section{Introduction}

An important and singular air pollution problem is related with rocket launches. The burning of rocket engines during the first few seconds prior to and immediately following vehicles launchings results in the formation of a large cloud of hot, buoyant exhaust products near the ground level, which subsequently rises and entrains ambient air until the temperature and density of the cloud reach an approximate equilibrium with ambient conditions. The United States (US) space activities are conducted by the military personnel (Air Force) and civilians (NASA). The US Air Force have 2 rocket launching centers, being one at East Coast (Cape Canaveral Air Force Base in Florida) and other one at west part (Vandenberg Air Force Base in California). These launching centers are close to 
big and populated region/cities that may be affected by the gases released during the launchings. In order to estimate the risks associated and the environmental impacts from the launchings (either normal or failed), a special model named REEDM (Rocket Exhaust Effluent Diffusion Model) was developed by Bjorklund et al. [1]. This model assumes a constant wind profile and Gaussian plume turbulence to access the movement of the clouds derived from the exhausted gases.

Unfortunately, there is no model fully ready to meet these demands in Brazil, or some experimental data dispersion of contaminants related to Alcântara Launch Center (ALC). Therefore, researches are very important and the developing of a system modeling designed to calculate peak concentration, dosage and deposition (resulting from both gravitational settling and precipitation scavenging) downwind from normal and aborted launchings to use in mission planning activities and environmental assessments, pre-launch forecasts of the environmental effects of launch operations and post-launch environmental analysis in Brazilian site. To this end, this paper aims to provide a framework that will allow the development of a model that considers the Brazilian site characteristics. The model is based on semi-analytical solution of the time dependent three-dimensional advection-diffusion equation. Furthermore, in the present work we report numerical simulations with Model Simulator of Rocket Effluent Dispersion (MSRED) approach using micrometeorological parameters and wind profile generated by Weather Research and Forecasting (WRF) model in the area around the ALC. Similarly to the REEDM model, the MSRED model calculates atmospheric pollutants concentrations based on vehicle emission, meteorological, and launch scenario data. Although based on relatively simple atmospheric dispersion physics, the code is complex, with a large number of variables. Therefore, firstly, our main intention in this work is to show the construction of the mathematical model for rocket effluent dispersion.

To reach the objective we organize the paper as follows: in section 2, we report the physical approach with the theoretical basic assumptions. In section 3, we show the mathematical model with the solution of the advection-diffusion equation. In section 4, we show the turbulent parameterizations. In section 5 , are presented numerical results, and finally, in section 6 , the conclusions.

\section{Theoretical basic assumptions}

The initial problem, whose solution is particularly important for predicting the atmospheric dispersion of gases from static test firings launches, and other hot releases, is defining the source; i.e. the initial distribution of the gases resulting from the buoyancy of the hot exhaust gases. Observation shows that the exhaust gases form a cloud elevated above the surface. A combination of theoretical analysis and empirical observations has been used to create a mathematical model of the cloud and thus to provide a source description for subsequent atmospheric dispersion analyses. In addition to the meteorological parameters, which are the principal factors determining the turbulent diffusivity of the atmosphere, the depth of the surface transport layer or the presence of an inversion layer can profoundly affect the predicted ground-level concentrations of rocket exhaust gases. It is 
assumed that no transport of effluents occurs across the boundaries of a transport layer; hence, the effluents are trapped within their respective transport layers. Consequently, an interaction exists between the height of the surface transport layer and the height of the exhaust cloud stabilization in determining the downwind ground-level concentrations of exhaust gases. Although the amount of exhaust products contained in the ground cloud is a function of the local meteorology (principally the depth of the atmospheric boundary layer (ABL)), it is typically calculated to be that due to approximately the first $20 \mathrm{~s}$ of burn time of the space shuttle engines. This assumption is arrived at by considering the ground cloud to be formed by the exhaust cloud emitted through the flame trench for the first $10 \mathrm{~s}$ after ignition plus the column of exhaust products formed during the following $10 \mathrm{~s}$.

The space shuttle flight system will be powered by chemical solid rocket motors and liquid rocket engines. The main environmental effect at launch arises from combustion of the space shuttle SRM's (Solid Rocket Motors). Combustion products are released into various layers of the atmosphere as the vehicle gains altitude during launch. The bulk of the shuttle combustion products are released into the troposphere. In the middle and upper troposphere, the exhaust products are deposited in a thin column because of the relatively high velocity of the vehicle there. This column quickly mixes and dissipates. At lower altitudes (near the surface) a cloud of exhaust products is generated. This "ground-cloud" disperses slowly and has been the subject of extensive analysis [1]. In a normal launch, the ground-cloud is formed at the base of the launch platform; it includes hot exhaust products from the SRM's, the main liquid propulsion engines, steam from launch platform cooling and acoustic damping water injection, and some sand and dust drawn into the cloud from the platform area [2]. Because of the high temperature of the gas cloud, buoyancy effects cause it to rise to an altitude of 0.7 to $3 \mathrm{~km}$, where it stabilizes because of the cooling of the gases.

A tool for analysis of toxic dispersion in the US and to support the release and evaluation of public risk is the REEDM model $[1,3,4]$. Therefore, this program was used as reference for modeling physics and mathematics of our problem and, consequently, in the development of the 3D-MSRED program. For more details about this approach see Bjorklund et al. [1]. It is important to mention that the REEDM model does not account for atmospheric chemical reactions of the launch cloud's toxic species. For example, the model assumes that all Hydrochloric acid $(\mathrm{HCl})$ emitted remains in the cloud as gaseous $\mathrm{HCl}$. There are important toxic removal processes occurring in the clouds that will reduce toxic ground-level concentrations. Furthermore, the model assumes that all chemical combustion reactions are completed before the cloud rise process takes place and, therefore, does not attempt to recompute chemical composition and additional heat release during the cloud rise computations. Input parameters that entail significant uncertainty were treated in a conservative fashion in the sense that choices were made to favor overestimating rather than underestimating the toxic chemical concentrations being evaluated for the environmental assessment study. Some vehicle data is easily obtained and verified, such as the stage propellant types, quantities and burn rates. Other input parameters required by model are based on 
derived values obtained from mathematical and physical models, empirical measurement data or engineering judgment from the vehicle designer or range safety experts.

The main assumption used in the REEDM model about the nature and behavior of the cloud released by the rocket is that it can be initially defined as a single cloud that grows and moves, but remains as a single cloud during the formation of the ascending phase of it. This concept is illustrated in Figure 1, and can be noticed that the model is designed for REEDM concentrations from the vertical position of the stabilized cloud.

The aspect "multilayer" is still used in the REEDM model and relates to the partitioning of cloud stabilized in "disks" of material from the cloud represented by different meteorological levels at different altitudes [6]. Typical levels are 20 to $50 \mathrm{~m}$ deep. Since the cloud is defined and has reached the condition of thermal stability with the atmosphere, the cloud is partitioned into "disks". The position of each disk with respect to the origin (launch pad) is determined based on the rise time of the cloud through a sequence of layers that are defined using meteorological measuring levels obtained from a radiosonde. Each layer can have a single meteorological speed and wind direction that moves the disk into the same cloud.

The hypothesis of transport in a straight line used in the REEDM model during the transport of clouds and phase dispersion ignores the possibility of wind fields that can arise in complex mountainous terrain or may evolve during the passage of a sea breeze front or greater scale. Thus, it is recommended that the assumption of uniform wind is limited to the transport of the plume at distances not exceeding $25 \mathrm{~km}$. In this sense, the model does forecast REEDM concentration ranging from 5 to $10 \mathrm{~km}$ from the launch pad, so that this hypothesis is not a problem.

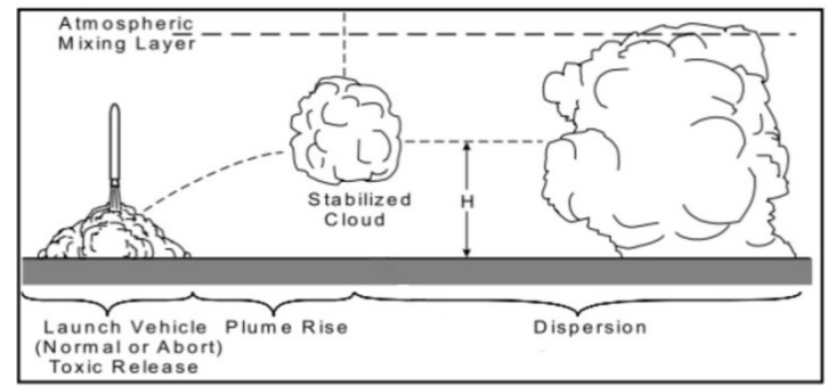

Figure 1: Conceptual illustration of cloud formation (source), "cloud-rise" and atmospheric dispersion of the cloud [5].

The REEDM model assumes that all chemical reactions are completed before the combustion process of the rise of the cloud. A mass fraction is assigned to each constituent and the total mass of the source (cloud) is multiplied by this fraction to determine the total mass of each chemical component in the cloud. The molecular weight of each species is used to convert the mass concentration per 
unit volume $\left(\mathrm{mg} / \mathrm{m}^{3}\right)$ to part per million ( $\left.\mathrm{ppm}\right)$. Besides, the model makes predictions instantaneous and average concentration in time (typically $10 \mathrm{~min}$ average). In many situations it is made an average of 1 hour to compute the average concentrations. A shorter average time is appropriate for exposure to the cloud of the rocket, because the source (cloud) typically goes on a receiver with a time scale of tens of minutes before the hour.

\section{The mathematical approach}

The advection-diffusion equation of air pollution in the atmosphere is essentially a statement of conservation of the suspended material. The concentration turbulent fluxes are assumed to be proportional to the mean concentration gradient which is known as Fick-theory. This assumption, combined with the continuity equation, leads to the advection-diffusion equation [7]:

$$
\frac{\partial c}{\partial t}+u \frac{\partial c}{\partial x}+v \frac{\partial c}{\partial y}+\left(w-v_{g}\right) \frac{\partial c}{\partial z}=\frac{\partial}{\partial x}\left(K_{x} \frac{\partial c}{\partial x}\right)+\frac{\partial}{\partial y}\left(K_{y} \frac{\partial c}{\partial y}\right)+\frac{\partial}{\partial z}\left(K_{z} \frac{\partial c}{\partial z}\right)-\lambda c-\Lambda c
$$

For $0<z<h, 0<y<L_{y}$ and $x>0$, where $c$ denotes the average concentration, $h$ is the ABL height, $L_{y}$ is far away from the source, $K_{x}, K_{y}, K_{z}$ and $u, v, w$ are the Cartesian components of eddy diffusivity and wind, respectively, $v_{g}$ is the gravitational settling, $\lambda$ represents a chemical-physical decay coefficient and $\Lambda$ is the scavenging coefficient. The decay term $\lambda$ represents in situ loss associated with processes such as chemical reaction or radioactive decay.

The $x$-axis of the Cartesian coordinate system is aligned in the direction of the actual wind near the surface, the $y$-axis is oriented in the horizontal crosswind direction, and the $z$-axis is chosen vertically upwards. In order to solve the Eq. (1) we include the following assumption: the lateral component of the mean flow is assumed to be zero $(v=0)$.

The mathematical description of the dispersion problem represented by the Eq. (1) is well posed when it is provided by initial and boundary conditions. Indeed, it is assumed that at the beginning of the pollutant release the dispersion region is not polluted, this means:

$$
c(x, y, z, 0)=0 \quad \text { at } t=0
$$

and a source of constant emission rate $Q$ is assumed:

$$
c(0, y, z, t)=\frac{Q}{u(z)}\left[\eta(\mathrm{t})-\eta\left(\mathrm{t}-\mathrm{t}_{\mathrm{r}}\right)\right] \delta\left(y-y_{o}\right) \delta\left(z-H_{s}\right) \quad \text { at } \quad x=0
$$

where $\delta$ is the Dirac delta function, $H_{s}$ the source height, $\eta$ is the Heaviside function and $t_{r}$ is the duration of release [8].

The pollutants are also subjected to the boundary conditions:

$$
K_{z} \frac{\partial c}{\partial z}=0 \quad \text { at } \quad z=h
$$


and

$$
K_{z} \frac{\partial c}{\partial z}=V_{d} c \quad \text { at } \quad z=0
$$

where $h$ is the ABL height and $V_{d}$ is the deposition velocity. In the $y$-direction, we have the conditions:

$$
\frac{\partial c}{\partial y}=0 \quad \text { at } \quad y=0, L_{y}
$$

In the following we assume that $K_{x}, K_{y}, K_{z}$ as well the wind speed $u$ depend only on the variable $z$ and we assume an averaged value ( $w$ and $v_{g}$ are constant). The stepwise approximation is applied in problem (1) by discretization the height $h$ into sub-layers in such manner that inside each sub-layer average values for $K_{x}$, $K_{y}, K_{z}$ and $u$ are taken. At this point it is important to remark that this procedure transforms the domain of problem (1) into a multilayered-slab in the $z$ direction. Furthermore, this approach is quite general in the sense it can be applied when these parameters are an arbitrary continuous function of the $z$ variable. Indeed, it is now possible to recast problem (1) as a set of advective-diffusive problems with constant parameters, which for a generic sub-layer reads like:

$$
\frac{\partial c_{n}}{\partial t}+u_{n} \frac{\partial c_{n}}{\partial x}+\left(w-v_{g}\right) \frac{\partial c_{n}}{\partial z}=K_{x}^{n} \frac{\partial^{2} c_{n}}{\partial x^{2}}+K_{y}^{n} \frac{\partial^{2} c_{n}}{\partial y^{2}}+K_{z}^{n} \frac{\partial^{2} c_{n}}{\partial z^{2}}-\lambda c_{n}-\Lambda c_{n}
$$

for $n=1: N$, where $N$ denotes the number of sub-layers and $c_{n}$ denotes the concentration at the $n^{\text {th }}$ sub-interval. Besides which, two boundary conditions are imposed at $z=0$ and $h$ given by equations (4) together with the continuity conditions for the concentration and flux of concentration at the interfaces must be considered, in order to be possible to uniquely determine the $2 \mathrm{~N}$ arbitrary constants appearing in the solution of the set of problems (6).

Now, we are in position of applying the GITT (Generalized Integral Transform Technique) method in the $y$-direction. The formal application of the GITT method [9] begins with the choice of the problem of associated eigenvalues (also known in the literature as the auxiliary problem) and their respective boundary conditions:

$$
\begin{array}{cr}
\psi_{i}^{\prime \prime}(\mathrm{y})+\lambda_{i}^{2} \psi_{i}(y)=0 & \text { at } \quad 0<y<L_{y} \\
\psi_{i}^{\prime}(y)=0 & \text { at } \quad y=0, L_{y}
\end{array}
$$

In accordance with the formalism of GITT, the first step is to expand the variable $c(x, y, z, t)$ into the following form:

$$
c_{n}(x, y, z, t)=\sum_{i=0}^{\infty} \frac{\bar{c}_{n i}(x, z, t) \psi_{i}(y)}{\mathrm{N}_{i}^{1 / 2}}
$$


Following the steps of the work of Costa et al. [10] we obtain the well-known solution, where the concentration is obtained by inverting numerically the transformed concentration $\widehat{c}_{n i}$ by a Gaussian quadrature scheme:

$$
\begin{aligned}
& \bar{c}_{n i}(x, z, t)=\sum_{j=1}^{M_{1}} \frac{p_{j}}{t} a_{j} \sum_{k=1}^{M_{2}} \frac{p_{k}}{x} a_{k}\left[G_{1} e^{\left(R_{1}+R_{2}\right) z}+G_{2} e^{\left(R_{1}-R_{2}\right) z}+\right. \\
& \left.\frac{\left(1-e^{-\frac{p_{j}}{t} t_{r}}\right)}{\left(p_{j} / t\right)} \frac{Q}{R_{3}}\left(e^{\left(R_{1}-R_{2}\right)\left(z-H_{s}\right)}-e^{\left(R_{1}+R_{2}\right)\left(z-H_{s}\right)}\right) H\left(z-H_{s}\right)\right]
\end{aligned}
$$

where

$$
\begin{gathered}
R_{1}=\frac{1}{2} \frac{\left(w-v_{g}\right)}{K_{z}} ; R_{2}=\frac{1}{2}\left(\frac{p+\lambda+\Lambda+s u_{n} \beta+K_{y} \lambda_{i}^{2}}{K_{z}}\right)^{1 / 2} \\
R_{3}=\frac{N_{i}^{1 / 2} K_{z}}{\psi_{i}\left(y_{0}\right) \beta}\left[\left(\frac{w-v_{g}}{K_{z}}\right)^{2}+4\left(\frac{p_{j}}{t}+\lambda+\Lambda+\frac{p_{k}}{x} u_{n} \beta+K_{y} \lambda_{j}^{2}\right)\right]^{1 / 2} \\
\text { and } \beta=\left(1-\frac{K_{x} p_{k}}{u_{n} x}\right)
\end{gathered}
$$

and $H\left(z-H_{s}\right)$. The constants $a_{j}, a_{k}$ and $p_{j}, p_{k}$ are the weights and roots of the Gaussian quadrature scheme [11] while $j$ and $k$ are the quadrature points.

Finally, using the Eq. (8) we obtain:

$$
\begin{aligned}
& c_{n}(x, y, z, t)=\sum_{i=0}^{\infty} \frac{\psi_{i}(y)}{N_{i}^{1 / 2}}\left\{\sum _ { j = 1 } ^ { M _ { 1 } } \frac { p _ { j } } { t } a _ { j } \sum _ { k = 1 } ^ { M _ { 2 } } \frac { p _ { k } } { x } a _ { k } \left[G_{1} e^{\left(R_{1}+R_{2}\right) z}+G_{2} e^{\left(R_{1}-R_{2}\right) z}+\right.\right. \\
& \left.\left.\frac{\left(1-e^{-\frac{p_{j}}{t} t_{r}}\right)}{\left(p_{j} / t\right)} \frac{Q}{R_{3}}\left(e^{\left(R_{1}-R_{2}\right)\left(z-H_{s}\right)}-e^{\left(R_{1}+R_{2}\right)\left(z-H_{s}\right)}\right) H\left(z-H_{s}\right)\right]\right\}
\end{aligned}
$$

This equation is then truncated for a sufficiently large number of series summation terms in order to obtain the final solution for the problem (6). Therefore, after determining the stabilization time and the source (multiple sources due to partitioning of the cloud), the final ground-level concentration will be the contribution from all sources: 


$$
C(x, y, z, t)=\sum_{i}^{n} c_{i}(x, y, z, t), \quad \text { where } i=1,2,3, \ldots, n \text {. }
$$

where $n$ represents the $\mathrm{n}^{\text {th }}$ source due to the partitioning of the cloud of pollutants released by the rocket at the time of stabilization.

\section{Boundary layer parameterization}

In principle, diffusion of a cloud can be predicted mathematically. The problem of predicting the dispersion of a pollutant released in the atmosphere becomes primarily that of determining the proper diffusion coefficient. Generally this problem has been handled by defining broad classes of meteorological conditions (e.g., "stable", "neutral" and "unstable") and establishing empirical measures of the turbulent diffusion coefficient for each condition. However, if suitable meteorological measurements are available, these empirical measures can be related to more detailed features of the atmosphere.

In the atmospheric diffusion problems the choice of a turbulent parameterization represents a fundamental aspect for pollutant dispersion modeling. The reliability of each model strongly depends on the way the turbulent parameters are calculated and is related to the current understanding of the ABL [12]. In terms of the scaling parameters the vertical eddy diffusivity can be formulated as Degrazia et al. [13] (under convective conditions):

$$
\frac{K_{z}}{w_{*} h}=0.22\left(\frac{z}{h}\right)^{1 / 3}\left(1-\frac{z}{h}\right)^{1 / 3}\left[1-\exp \left(-\frac{4 z}{h}\right)-0.0003 \exp \left(\frac{8 z}{h}\right)\right]
$$

The micrometeorological parameters can be adapted from the routine of the model AERMET/AERMOD [14], whose function is to calculate the parameters $u *$ (friction velocity), $L$ (Monin-Obukhov length), $w *$ (convective velocity), $h$ (ABL height) and $H$ (heat flux) from the sounding (including the vertical wind speed) taken in ALC. However, in this work these parameters are derived from WRF mesoscale model.

\section{Numerical simulations}

The case study was modeled using the WRF Model version 3.5 [15] to generate meteorological fields in a four-day simulation, from March 18, 2013 at 00:00 h GMT to March 22, 2013 at 00:00h GMT. The horizontal resolution of the grid and its nests were $8.1 \mathrm{~km}, 2.7 \mathrm{~km}, 900 \mathrm{~m}, 300 \mathrm{~m}$ and $100 \mathrm{~m}$, and the horizontal dimensions, in grid cells, were $40 \times 40,64 \times 64,88 \times 88,76 \times 76$ and $112 \times 112$, for domain 1 to 5 , respectively, with 70 vertical levels. 
In order to run the WRF model to properly generate the meteorological fields, we used final analysis data from the Global Forecast System (GFS) with a resolution of 1 arc degree. Silva and Fisch [16] showed that the WRF model can be successfully applied to simulate the meteorological conditions of ALC region.

For this work, we chose to apply a large-eddy simulation in WRF to better represent the turbulence in the ALC region, activating the LES option available in WRF for real-world applications.

One of the major challenges needed to accomplish this task of running WRF in LES mode for real cases, was to get and configure a new and complete terrain dataset information for the ALC region in a very high resolution $(\sim 100 \mathrm{~m})$, since the highest resolution of the default WRF terrain dataset is $1 \mathrm{~km}$, which is not suitable to run large-eddy simulations. Thus, we downloaded from USGS site a GeoTIFF dataset of the terrain elevation for the surrounding area of the ALC site. Then, we processed this dataset, generating a new one in the geogrid format, enabling WRF to model this case using very high resolution terrain information of $100 \mathrm{~m}$.

Figure 2 presents two scenarios of the surface wind field simulated by the WRF model at the time of March 19, 2013 at 16:00 h GMT, and March 20, 2013 at 11:00 $\mathrm{h} \mathrm{GMT}$, for domain 5. From Figure 2, it is possible to note that the wind is predominantly blowing from the northeastern direction at the first scenario, flowing to the continent in the direction of an inhabited area, and a hypothetical launch at this time would not impact in populated areas, like the large city of São Luís. Some hours later the scenario changes: the wind direction starts to change, and, at March 20, 2013 at 11:00 h GMT, 19 hours later, the wind field entirely changes and blows from the southeastern direction, showing that in few hours the meteorological scenario can considerably change. Figure 2 has been produced using the program VAPOR [17].

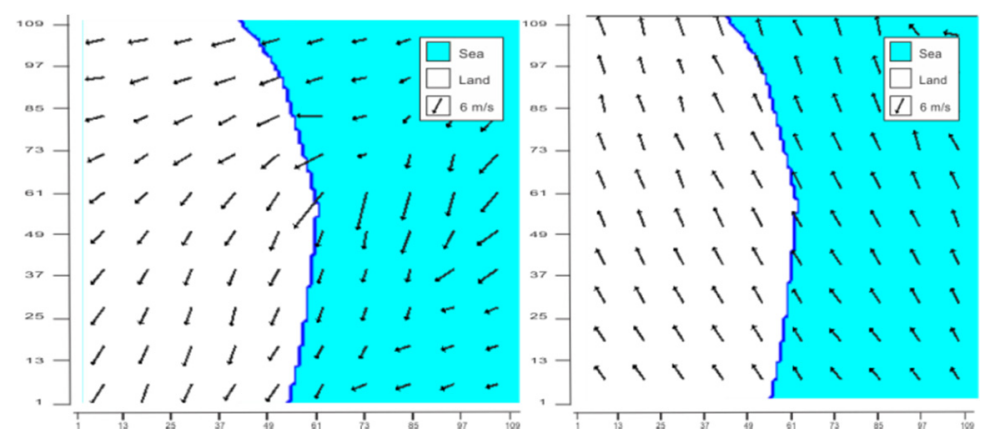

Figure 2: Simulated wind field (m/s) for 19 March 2013 at 16:00 h GMT (left), and 20 March 2013 at 11:00 h GMT (right), for domain 5.

Finally, we show a simulation considering a grid of $100 \times 100 \mathrm{~km}$ in the region covered by the ALC. The main points are shown in the Figure 3, which are shown the vector wind speed and dispersion of the plume. The concentration unit is ppm. 


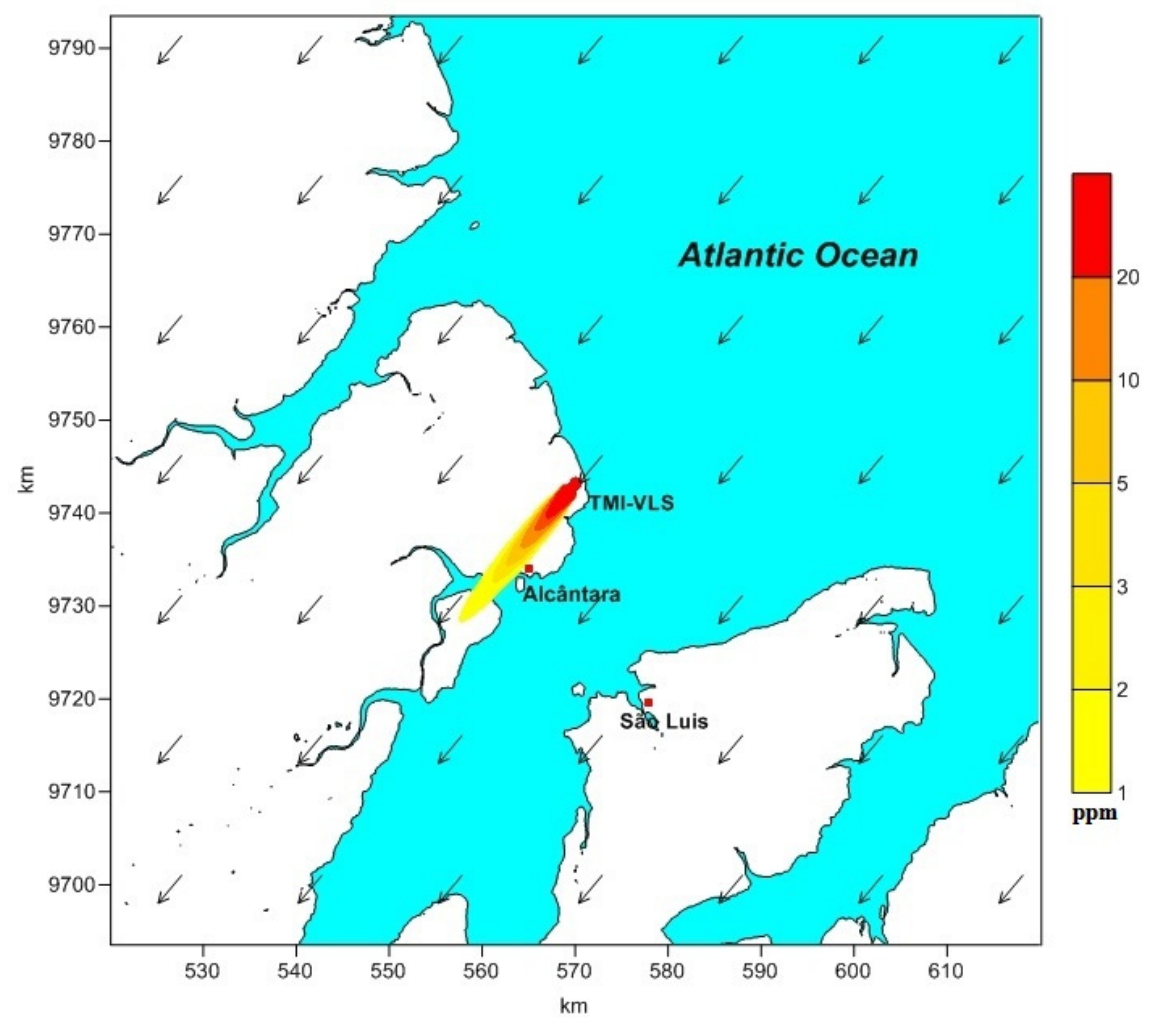

Figure 3: Plume generated in the simulation (region of $100 \times 100 \mathrm{~km}$ ). TMI represent the Tower Mobile Integration and VLS is the Satellite Launch Vehicle.

\section{Summary and conclusions}

A mathematical model has been developed which uses the characteristics of the rocket exhaust products and launch site meteorology to predict the rises, growth and dispersal of the ground cloud. This model considers the duration time release, chemical-physical decay, settling velocity, scavenging coefficient and can be applied for describing the turbulent dispersion of many scalar quantities, such as air pollution, radioactive material, heat, and so on. Due to lack of experimental data, we report numerical simulations with the approach using micrometeorological parameters and wind profile generated by WRF model in the area around the Alcântara Launch Center. From the previous results, we promptly notice the aptness this model to understand the time evolution of the concentration and its dependency on the duration of the contaminant emission. In fact, this model, allow us to simulate the continuous, short-term and instantaneous emissions. In particular, the model is suitable for an initial and rapid assessment 
of atmospheric dispersion under emergency conditions without sophisticated computing resources. The model can be used in different conditions of atmospheric stability, making it possible to predict or simulate the concentration in accordance with emergency plans and pre and post-launches for environmental management in situations of rocket launches in Alcântara Launch Center. However, these results show that there is a need for thorough checking of the model with experimental observations of concentrations and meteorological parameters, which are planned in the region.

\section{Acknowledgements}

The authors thank CNPq and CAPES for the partial financial support of this work.

\section{References}

[1] Bjorklund, J.R., Dumbauld, J.K, Cheney, C.S. and Geary, H.V., User's manual for the REEDM (Rocket Exhaust Effluent Diffusion Model) compute program. NASA contractor report 3646. NASA George C. Marshall Space Flight Center, Huntsville, AL, 1982.

[2] Stephens, J.B and Stewart, R.B., Rocket exhaust effluent modeling for tropospheric air quality and environmental assessment. National Aeronautics and Space Administration - NASA Technical Report, 1977.

[3] Bjorklund, J.R., User instructions for the Real-Time Volume Source Dispersion Model (RTVSM). H.E Cramer Company, Inc. Report TR-90374-02, prepared for U.S. Army Dugway Proving Ground, Dugway, UT, 1990.

[4] Bjorklund, J.R., Bowers, J.F., Dodd, G.C. and White, J.M., Open Burn/Open Detonation Dispersion Model (OBODM) user's guide. Volume II, Technical description. West desert test center, US Army Dugway proving ground, Dugway, Utah, 1998.

[5] Nyman, R.L., NASA Report: Evaluation of Taurus II Static Test Firing and Normal Launch Rocket Plume Emissions, 2009.

[6] Dumbauld, R.K., Bjorklund, J.R. and Bowers, J.F., NASA/MSFC multilayer diffusion models and computer program for operational prediction of toxic fuel hazards. NASA Contractor Report CR-129006, NASA George C. Marshall Space Flight Center, Huntsville, AL, 1973.

[7] Huang, C.H., A theory of dispersion in turbulent shear flow. Atmos. Environ. 13, 453-461, 1979.

[8] Bianconi, R. and Tamponi, M., A mathematical model of diffusion from a steady source of short duration in a finite mixing layer. Atmos. Environ. 27A, 5, 781-792, 1993.

[9] Cotta, R., Integral transforms in computational heat and fluid flow. CRC Press, Boca Raton, 352 pp, 1993.

[10] Costa, C.P., Vilhena, M.T., Moreira, D.M. and Tirabassi, T., Semianalytical solution of the steady three-dimensional advection-diffusion equation in the PBL. Atmospheric Environment 40, 5659-5669, 2006. 
[11] Stroud, A.H. and Secrest, D., Gaussian Quadrature Formulas, Englewood Cliffs, N.J., Prentice Hall, Inc., 1966.

[12] Mangia, C., Moreira, D.M., Schipa, I., Degrazia, G.A., Tirabassi, T. and Rizza, U., Evaluation of a new eddy diffusivity parameterisation from turbulent Eulerian spectra in different stability conditions. Atmos. Environ. 36, 67-76, 2002.

[13] Degrazia, G.A., Campos Velho, H.F. and Carvalho, J.C., Nonlocal exchange coefficients for the convective boundary layer derived from spectral properties. Contr. Atmos. Phys., 57-64, 1997.

[14] EPA-454/B-03-002, User's Guide for the AERMOD meteorological preprocessor (AERMET), 2004.

[15] Skamarock, W.C. and Klemp, J.B., A Time-Split Nonhydrostatic Atmospheric Model for Weather Research and Forecasting Applications. Journal of Computational Physics 227, 3465-3485, 2008.

[16] Silva, A.F.G. and Fisch, G., Avaliação do Modelo WRF para a Previsão do Perfil do Vento no Centro de Lançamento de Alcântara. Brazilian Journal of Meteorology, in press, 2014.

[17] Clyne, J., Mininni, P., Norton, A., and Rast, M., Interactive desktop analysis of high resolution simulations: application to turbulent plume dynamics and current sheet formation. New Journal of Physics 9, 301, 2007. 